\title{
Stranger things
}

\section{From a combined effort by theorists, materials scientists and spectroscopists, topology has moved from a purely mathematical idea to the realization of unique properties in several condensed matter systems.}

Topology is a branch of mathematics that studies the properties of space that are invariant under continuous deformation (stretching, shrinking or bending is allowed, but cutting or gluing is not). It leads to the classification of spaces into different families: for instance, a sphere and a cube belong to the same topological family because they can be transformed into each other; a doughnut is not, because the creation of the inner hole is not allowed. Instead, it belongs to a different topological clan along with mugs (pictured).

Despite mathematical interest, topology only sneaked into physics and, in particular, materials science relatively recently. In systems with periodic symmetries such as crystals, the electronic properties can be understood by studying the effect of periodicity in the wavefunctions in reciprocal space ${ }^{1}$. In contrast, topological materials exhibit phases where the electronic properties are determined by topology rather than by local symmetry-breaking mechanisms. The topological properties of the electrons' wavefunctions are global properties of the material; this leads to remarkable transport properties that are robust up to a certain amount of defects, making these materials promising for technological applications.

David Thouless, Duncan Haldane and Michael Kosterlitz pioneered the study of the topological properties of quantum states, deservedly winning the 2016 Nobel Prize in Physics for their contributions. The first topologically nontrivial state of matter, the quantum Hall effect, was reported in 1980, with Klaus von Klitzing winning the 1985 Nobel Prize in Physics for its experimental observation. At low temperature and under the application of strong magnetic fields perpendicular to the sample, the Hall conductivity of a two-dimensional electron gas becomes quantized, taking values that are integer multiples of $e^{2} / h$ (where $h$ is Planck's constant and $e$ is the charge of an electron). In this state only the edges of the material conduct, and scattering is suppressed because the electrons cannot reverse their direction; in order to do so they would have to jump to the opposite edge, which is prevented by a macroscopic separation between them. The transport properties of the edge states do not depend on the particular geometry of the sample, and they are topologically protected ${ }^{2}$. In their Perspective in this issue, Jing Wang and Shou-Cheng Zhang discuss how the theoretical predictions, combined with progress in materials synthesis and characterization techniques, led to the recent explosion of topological studies in condensed matter ${ }^{3}$.

One of the most exciting research directions is the study of topological insulators (or quantum spin Hall states), and Weyl and Dirac semimetals ${ }^{4}$. These materials are characterized by the existence of topologically protected points or nodes where the valence and conduction bands touch at the Fermi energy, enabling the existence of excitations that behave as fermions with unique properties. For instance, Weyl fermions have been observed as excitations in weakly correlated electron systems with inversion-symmetry breaking. It has also been predicted that they can be realized in magnetically ordered states in correlated materials exhibiting spontaneously time-reversal-symmetry breaking. In this issue, Satoru Nakatsuji and colleagues report experimental evidences for magnetic Weyl fermions in a non-collinear antiferromagnet, which could lead to the control of the characteristic topological Weyl points by applying magnetic fields ${ }^{5}$.

In addition to theoretical predictions, a driving force for the discovery of new states of matter has been the development of experimental techniques that allow better control of interfaces, crystal quality and thickness of the materials, as well as their characterization. For instance, the improvement of the quality of the material systems exhibiting the quantum Hall effect led to the discovery of the fractional quantum Hall effect (awarded the 1998 Nobel Prize in Physics). Nitin Samarth reviews how the improvement in deposition techniques of thin films has impacted the discovery and development of quantum materials ${ }^{6}$ (those whose properties cannot be explained through classical physics, although they do not necessarily stem from topological properties). Going one step further, Dmitri Basov, Richard Averitt and David Hsieh discuss in their Review how the properties of quantum materials can be controlled ${ }^{7}$. Finally, Kathryn Moler showcases in a Commentary the available experimental techniques for

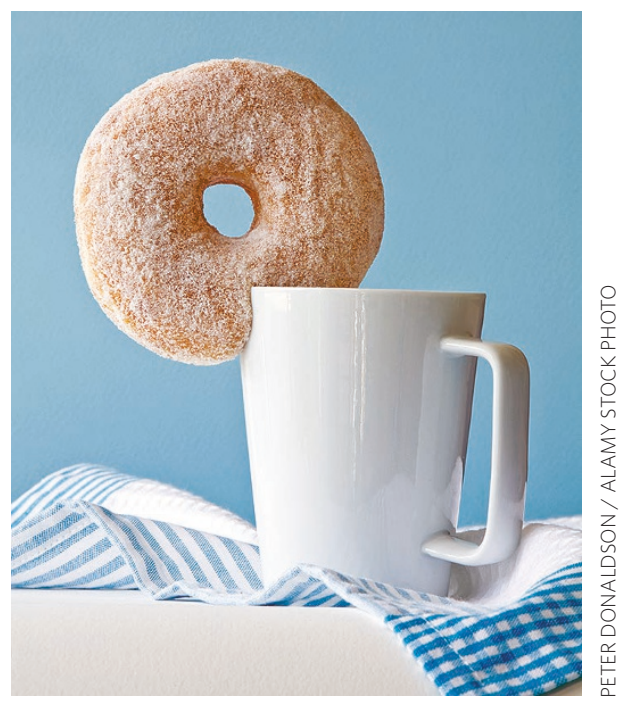

their study ${ }^{8}$. These pieces are complemented by two Reviews and a Commentary on the physics, applications and characterization of the photoemission properties of quantum materials, which appear in the November issue of Nature Physics. All together these constitute a joint Insight on quantum materials (https://www.nature.com/ collections/quantum-materials), which we will distribute as a separate supplement thanks to the support of the Gordon and Betty Moore Foundation, and the Simons Foundation.

Topological matter is a fertile and active research field for the observation of unique quantum properties at the macroscale, as well as the understanding of fundamental aspects of quantum mechanics. The synthesis of new materials combined with novel characterization techniques will hopefully lead to technological applications. Beyond such tangible outcomes, the long and unexpected path topology has taken since the discovery of the quantum Hall effect should be a constant reminder that things can always get stranger and more exciting at any given moment.

\footnotetext{
References

1. Asorey, M. Nat. Phys. 12, 616-618 (2016).

2. Thouless, D. J., Kohmoto, M., Nightingale, M. P. \& den Nijs, M Phys. Rev. Lett. 49, 405-408 (1982).

. Wang, J. \& Zhang, S.-C. Nat. Mater. 16, 1062-1067 (2017).

4. Burkov, A. A. Nat. Mater. 15, 1145-1148 (2016).

5. Kuroda, K. et al. Nat. Mater. 16, 1090-1095 (2017).

6. Samarth, N. Nat. Mater. 16, 1068-1076 (2017).

7. Basov, D. N., Averitt, R. D. \& Hsieh, D. Nat. Mater. 16, 1077-1088 (2017).

8. Moler, K. A. Nat. Mater. 16, 1049-1052 (2017).
} 